\title{
Systematic uncertainties in the precise determination of the strangeness magnetic moment of the nucleon
}

\author{
D.B. Leinweber ${ }^{1,2}$, S. Boinepalli ${ }^{1}$, A.W. Thomas ${ }^{1,2}$, A.G. Williams ${ }^{1}$, R.D. Young ${ }^{1,2}$, J.B. Zhang ${ }^{1}$, and J.M. Zanotti ${ }^{3}$ \\ 1 Special Research Center for the Subatomic Structure of Matter, and Department of Physics, University of Adelaide, \\ Adelaide SA 5005, Australia \\ 2 Jefferson Lab, 12000 Jefferson Ave., Newport News, VA 23606, USA \\ 3 John von Neumann-Institut für Computing NIC, Deutsches Elektronen-Synchrotron DESY, D-15738 Zeuthen, Germany
}

Received: 1 December 2004 / Published Online: 8 February 2005

(C) Società Italiana di Fisica / Springer-Verlag 2005

\begin{abstract}
Systematic uncertainties in the recent precise determination of the strangeness magnetic moment of the nucleon are identified and quantified. In summary, $G_{M}^{s}=-0.046 \pm 0.019 \mu_{N}$.
\end{abstract}

PACS. 13.40.Em Electric and magnetic moments - 12.38.Gc Lattice QCD calculations - 12.39.Fe Chiral Lagrangians

\section{Introduction}

Recent low-mass lattice QCD simulation results combined with new chiral extrapolation techniques and the principle of charge symmetry have enabled a precise determination of the strangeness magnetic moment of the nucleon $G_{M}^{s}$ [1]. In this paper, the systematic errors of the approach are explored and quantified. In particular, we examine the sensitivity of $G_{M}^{s}$ and the magnetic moments of the baryon octet to the regulator-mass scale of finiterange regularized chiral effective field theory, the lattice scale determination, the finite-volume of the lattice, and the quenched approximation.

\section{Charge symmetry}

The approach 1 centres around two equations for the strangeness magnetic moment of the nucleon, $G_{M}^{s}$, obtained from charge symmetry

$$
\begin{aligned}
G_{M}^{s} & =\left(\frac{{ }^{\ell} R_{d}^{s}}{1-{ }^{\ell} R_{d}^{s}}\right)\left[2 p+n-\frac{u^{p}}{u^{\Sigma}}\left(\Sigma^{+}-\Sigma^{-}\right)\right], \\
G_{M}^{s} & =\left(\frac{{ }^{\ell} R_{d}^{s}}{1-{ }^{\ell} R_{d}^{s}}\right)\left[p+2 n-\frac{u^{n}}{u^{\Xi}}\left(\Xi^{0}-\Xi^{-}\right)\right] .
\end{aligned}
$$

Here the baryon labels represent the experimentally measured baryon magnetic moments and ${ }^{\ell} R_{d}^{s} \equiv G_{M}^{s} /{ }^{\ell} G_{M}^{d}$ is the ratio of $s$ - and $d$-quark sea-quark loop contributions, depicted in the right-hand diagram of Fig. 1. ${ }^{\ell} R_{d}^{s}$ lies in the range $(0,1)$. The ratios $u^{p} / u^{\Sigma}$ and $u^{n} / u^{\Xi}$ are ratios of valence-quark contributions to baryon magnetic moments in full QCD as depicted in the left-hand diagram of Fig. 1 The latter are determined by lattice QCD calculations [1, 3, 4,56$]$.
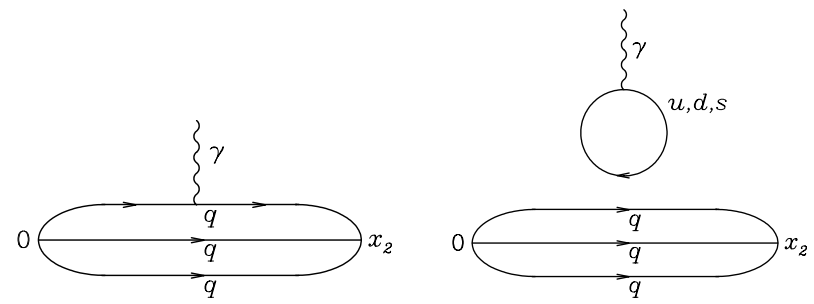

Fig. 1. Diagrams illustrating the two topologically different insertions of the current within the framework of lattice QCD. These skeleton diagrams for the connected (left) and disconnected (right) current insertions are dressed by an arbitrary number of gluons and quark loops

Equating (11) and (2) provides a linear relationship between $u^{p} / u^{\Sigma}$ and $u^{n} / u^{\Xi}$ which must be obeyed within QCD under the assumption of charge symmetry - itself typically satisfied at the level of $1 \%$ or better 2 . There are no other systematic uncertainties associated with this constraint. Figure 2 displays this relationship by the dashed and solid line. Since this line does not pass through the point $(1.0,1.0)$, corresponding to the simple quark model assumption of universality, there must be an environment effect exceeding $12 \%$ in both ratios or approaching $20 \%$ or more in at least one of the ratios. To determine the sign of $G_{M}^{s}$, it is sufficient to determine where on this constraint curve, QCD resides.

\section{$3 u^{p} / u^{\Sigma}$ and $u^{n} / u^{\Xi}$ determination}

Our present precise analysis has been made possible by a significant breakthrough in the regularization of the chiralloop contributions to hadron observables $[7,8,9,9$. Through 


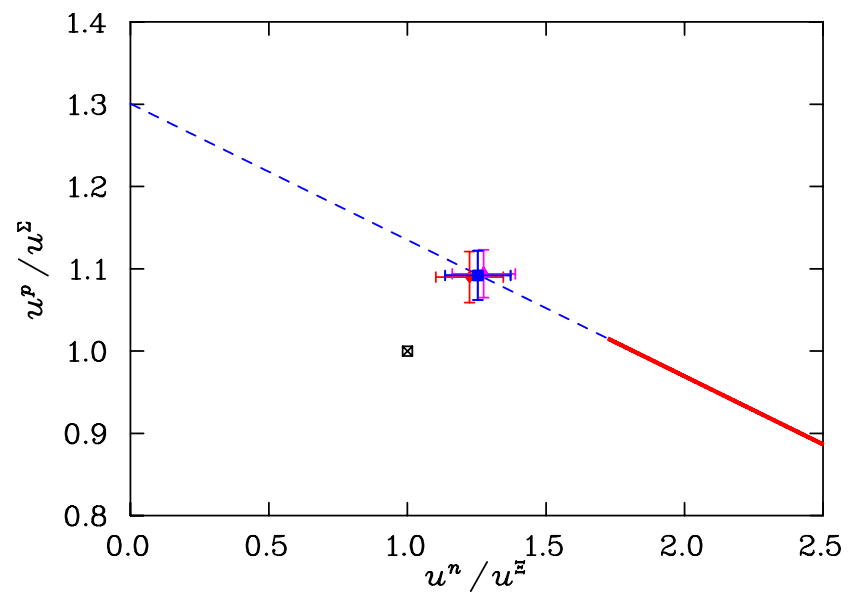

Fig. 2. The straight line $\left(\right.$ dashed $G_{M}^{s}(0)<0$, solid $\left.G_{M}^{s}(0)>0\right)$ indicates the constraint on the ratios $u^{p} / u^{\Sigma}$ and $u^{n} / u^{\Xi}$ implied by charge symmetry and the experimentally measured magnetic moments. The assumption of environment independent quark moments is indicated by the square. The dependence of the extrapolated ratios from lattice QCD simulations on the parameter $\Lambda=0.7,0.8$ and $0.9 \mathrm{GeV}$, (governing the size of pion-cloud corrections) is illustrated by the cluster of points with $\Lambda$ increasing from left to right

the process of regulating loop integrals via a finite-range regulator (FRR) [8,10], the chiral expansion is effectively re-summed to produce an expansion with vastly improved convergence properties.

The chiral expansion for the $u$-quark contribution to the proton magnetic moment in quenched QCD (QQCD), has the form

$$
\begin{aligned}
u^{p}= & a_{0}^{\Lambda}+u^{p} \chi_{\eta^{\prime}} I_{\eta^{\prime}}\left(m_{\pi}, \Lambda\right)+\chi_{\pi B} I_{B}\left(m_{\pi}, \Lambda\right) \\
& +\chi_{K B} I_{B}\left(m_{K}, \Lambda\right)+a_{2}^{\Lambda} m_{\pi}^{2}+a_{4}^{\Lambda} m_{\pi}^{4},
\end{aligned}
$$

where the repeated index, $B$, sums over allowed baryon octet and decuplet intermediate states. The dependence of the unrenormalized coefficients, $a_{i}^{\Lambda}$, and the associated dipole-vertex regulated loop integrals, $I\left(m_{\pi}, \Lambda\right)$, on the regulator parameter, $\Lambda$, is emphasized by the explicit appearance of $\Lambda$. The loop integrals are defined as

$$
\begin{aligned}
& I_{B}(m, \Lambda)= \\
& \quad-\frac{2}{3 \pi} \int d k \frac{\left(2 \sqrt{k^{2}+m^{2}}+\Delta_{B N}\right) k^{4} u^{2}(k, \Lambda)}{\left(k^{2}+m^{2}\right)^{3 / 2}\left(\sqrt{k^{2}+m^{2}}+\Delta_{B N}\right)^{2}}, \\
& I_{\eta^{\prime}}\left(m_{\pi}, \Lambda\right)=-\int_{0}^{\infty} d k \frac{k^{4}}{\left(k^{2}+m_{\pi}^{2}\right)^{\frac{5}{2}}} u^{2}(k, \Lambda),
\end{aligned}
$$

where $\Delta_{B N}$ is the relevant baryon mass splitting and the function $u(k, \Lambda)$ is the dipole-vertex regulator. The coefficients, $\chi$, denote the known model-independent coefficients of the non-analytic terms for $\pi$ and $K$ mesons in QQCD [11, 12.

Figure 3 illustrates a fit of FRR, quenched chiral perturbation theory $(\chi \mathrm{PT})$ to our fermion lattice results (solid curve), where only the discrete momenta allowed in the finite volume of the lattice are summed in evaluating the chiral loop integrals. The long-dashed curve that also

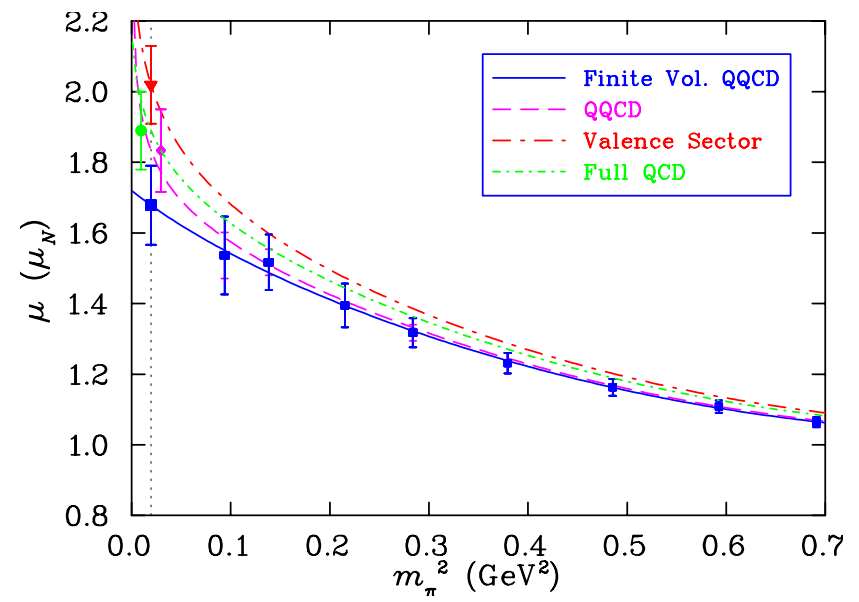

Fig. 3. The contribution of a single $u$ quark (with unit charge) to the magnetic moment of the proton. Lattice simulation results (square symbols for $m_{\pi}^{2}>0.05 \mathrm{GeV}^{2}$ ) are extrapolated to the physical point (vertical dashed line) in finite-volume QQCD as well as infinite volume QQCD. Estimates of the valence $u$ quark contribution in full QCD and the full $u$-quark sector contribution in full QCD are also illustrated. Extrapolated values at the physical pion mass (vertical dashed line), are offset for clarity

runs through the lattice results corresponds to replacing the discrete momentum sum by the infinite-volume, continuous momentum integral. For all but the lightest quark mass, finite volume effects are negligible.

Having determined the analytic coefficients $a_{0,2,4}^{\Lambda}$ for a particular choice of $\Lambda$, one can correct the chiral properties of the pion-cloud contribution from QQCD to full QCD 9 , 13 by changing the coefficients of the loop integrals, $\chi_{\eta^{\prime}}$, $\chi_{\pi B}, \chi_{K B}$ of (3), to their full QCD counter parts [11, 12. Valence quark contributions in full QCD are indicated by the long-dash-dot curve in Fig. 3 (i.e. sea-quark loop charges are zero) and the full $u$-quark sector including the $u$-sea-quark loop contributions are indicated by the short-dash-dot curve for $\Lambda=0.8 \mathrm{GeV}$. Figures 45 and 6 show similar results for the $u$ quark in $n, \Sigma^{+}$, and $\Xi^{0}$ respectively.

The importance of correcting for both finite-volume and quenching artifacts is illustrated in Figs. 7 and 8 , where the one standard deviation agreement between the chirally corrected lattice QCD simulation results and the experimentally measured baryon magnetic moments is highlighted.

\section{Systematic errors}

\subsection{Regulator dependence}

It is important to investigate systematic errors associated with the regulator-mass dependence of FRR $\chi \mathrm{PT}$. The extrapolated results of finite-volume quenched chiral effective field theory should be insensitive to the choice of regulator parameter. When working to sufficient order in 


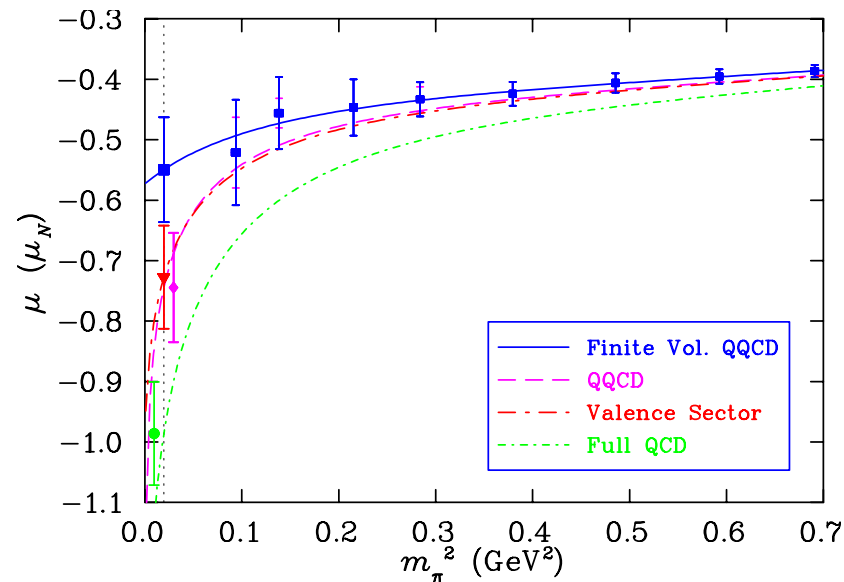

Fig. 4. The contribution of the $u$ quark (with unit charge) to the magnetic moment of the neutron. Curves and symbols are as described in Fig. 3

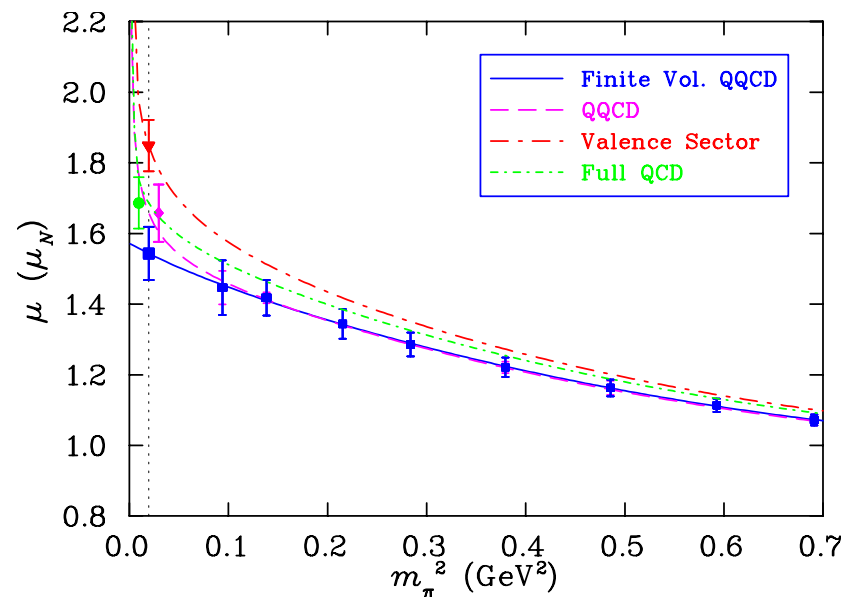

Fig. 5. The contribution of a single $u$ quark (with unit charge) to the magnetic moment of $\Sigma^{+}$. Curves and symbols are as for Fig. 3

the chiral expansion, changes in the regulation of loop integrals should be absorbed by changes in the unrenormalized coefficients, $a_{0,2,4}^{\Lambda}$, in a manner which preserves the invariant renormalized coefficients. The latter are reflected in Fig. 9 which illustrates the insensitivity of quenched baryon magnetic moments in a finite-volume to the regulator parameter $\Lambda$. This systematic error is small relative to the statistical error.

Since the finite-volume and quenching corrections are applied only to the loop integral contributions, the final results are $\Lambda$ dependent. In this case, the regulator of the loop integral has become a model for the axial-vector form factor of the nucleon, describing the coupling of pions to a core described by the analytic terms of the FRR expansion. This approach describes the relation between quenched and full QCD $N$ and $\Delta$ mass simulation results as a function of $m_{\pi}$ very accurately [13]. Figures 10] and 11] display results for positive and negative baryon magnetic moments in full QCD respectively. One-standard deviation agreement is achieved for $0.7 \leq \Lambda \leq 0.9$.

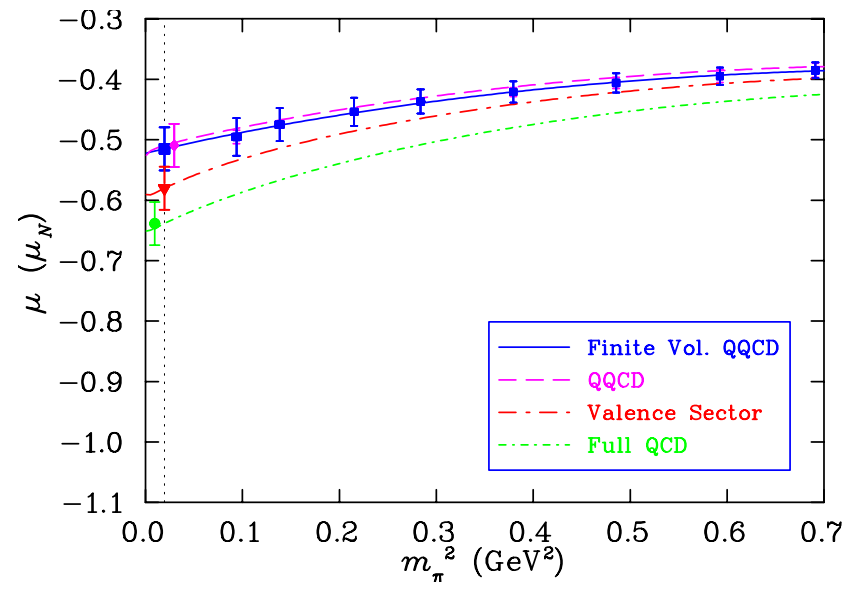

Fig. 6. The contribution of the $u$ quark (with unit charge) to the magnetic moment of the $\Xi^{0}$ hyperon. Curves and symbols are as described in Fig. 3

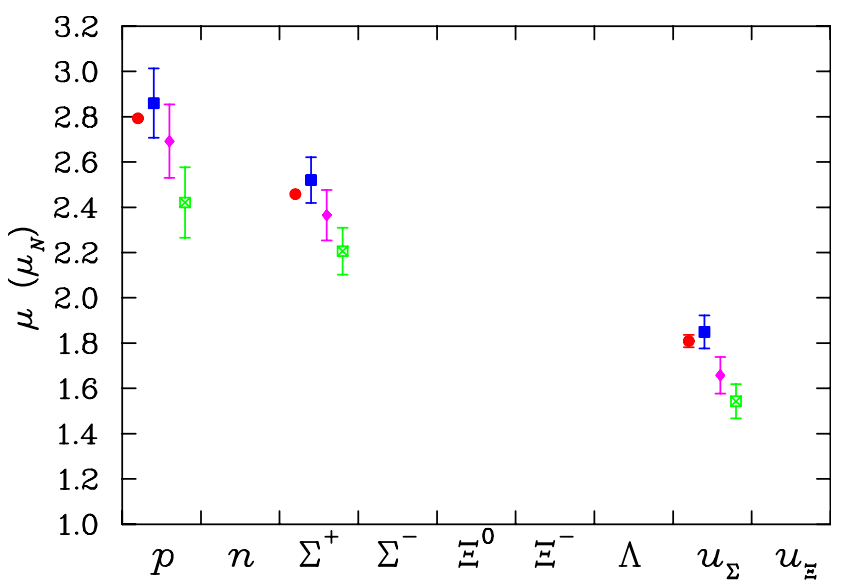

Fig. 7. The one standard deviation agreement between the chirally corrected lattice QCD simulation results (square symbols) and the experimentally measured baryon magnetic moments (circular symbols) having positive values. Finite-volume quenched results (crossed boxes) and infinite-volume quenched results (diamonds) are also illustrated to highlight the importance of correcting for both finite-volume and quenching artifacts

Because the strangeness magnetic moment of (1) and (2) depends only on ratios of magnetic moments, most of this $\Lambda$ dependence cancels in the final ratios, as illustrated by the close clustering of points in Fig. 2 ,

\subsection{Role of the decuplet in $\chi \mathrm{PT}$}

It is often argued that next-to-leading-order non-analytic (NLNA) contributions from the baryon decuplet are essential in describing the mass dependence of nucleon magnetic moments. While the decuplet baryon contributions are not necessarily small, we find the non-analytic curvature induced by these contributions is sufficiently subtle that it may be accurately absorbed by the analytic terms 


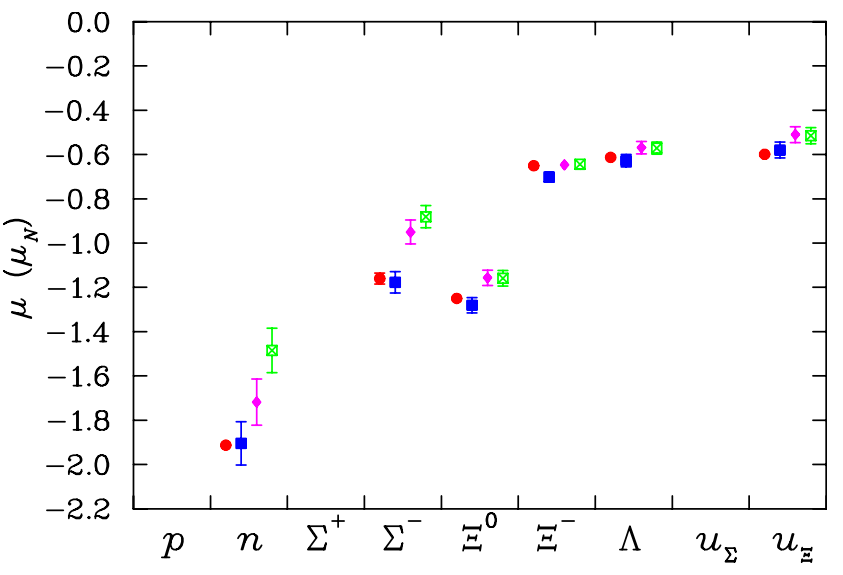

Fig. 8. The one standard deviation agreement between the chirally corrected lattice QCD simulation results and the experimentally measured baryon magnetic moments having negative values. Symbols are as in Fig. 7

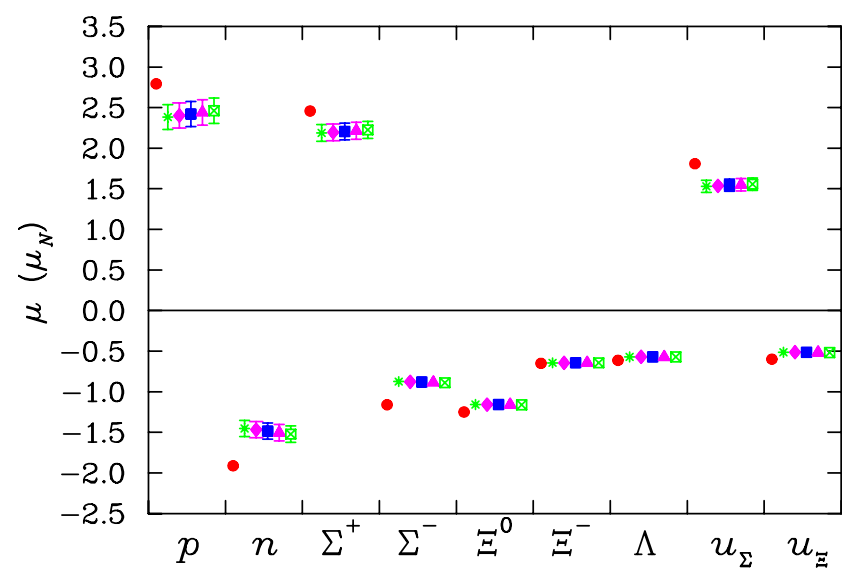

Fig. 9. The FRR $\chi \mathrm{PT}$ regulator mass dependence of finitevolume quenched chiral effective field theory. Experimental measurements are illustrated at left for each baryon for reference. Results for $\Lambda=0.6,0.7,0.8,0.9$ and $1.0 \mathrm{GeV}$ are illustrated from left to right for each baryon. The small systematic dependence on $\Lambda$ relative to the statistical error bars illustrated indicate the order of the chiral expansion is adequate for this analysis

of the chiral expansion. Figure12 illustrates the insensitivity of finite-volume quenched chiral effective field theory to NLNA decuplet-baryon contributions.

Figure 13 confirms that the NLNA decuplet-baryon contributions are indeed large in some cases and as such are important in correcting the artifacts of the quenched approximation. However, other more highlyexcited baryon resonances have small couplings to the ground-state baryon octet relative to that for the decuplet and provide negligible corrections.

\subsection{Scale dependence}

Setting the scale in quenched QCD simulations is somewhat problematic. Different observables lead to different

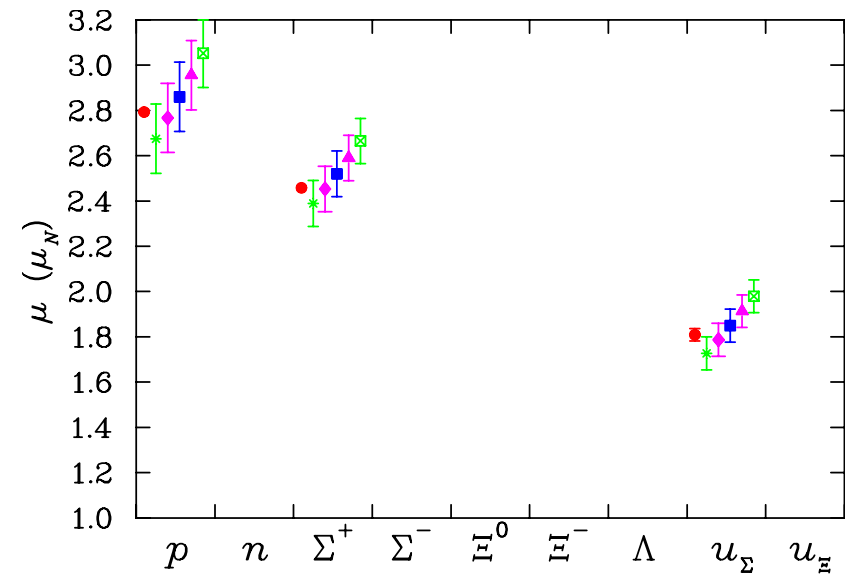

Fig. 10. The dependence of positive octet-baryon magnetic moments on the parameter $\Lambda=0.6,0.7,0.8,0.9$ and $1.0 \mathrm{GeV}$, governing the size of pion-cloud corrections associated with the finite-volume of the lattice and artifacts of the quenched approximation. Experimental measurements, illustrated at left by the filled circle for each baryon, indicate that optimal corrections are obtained for $0.6 \leq \Lambda \leq 0.9 \mathrm{GeV}$

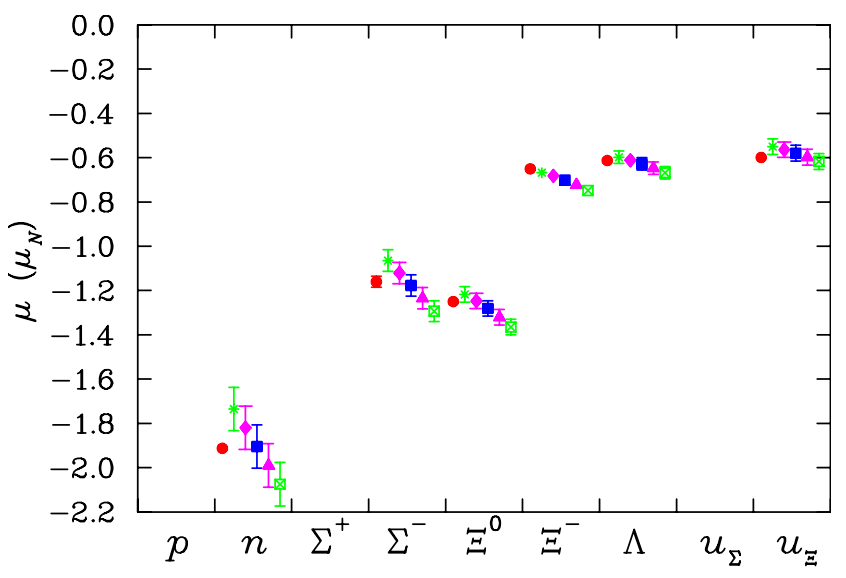

Fig. 11. The dependence of negative octet-baryon magnetic moments on the parameter $\Lambda=0.6,0.7,0.8,0.9$ and $1.0 \mathrm{GeV}$, governing the size of pion-cloud corrections associated with the finite-volume of the lattice and artifacts of the quenched approximation. Experimental measurements, illustrated at left by the filled circle for each baryon, indicate that optimal corrections are obtained for $0.7 \leq \Lambda \leq 0.9 \mathrm{GeV}$

lattice spacings, $a$. If one is explicitly correcting the oneloop pion-cloud contributions to hadronic observables, as we are here, then clearly one must set the scale using an observable insensitive to chiral physics. This excludes observables such as the rho-meson mass, nucleon mass, or the pion decay constant commonly used in the literature to hide the artifacts of the quenched approximation. On the other hand, the heavy-quark phenomenology of the static-quark potential provides an optimal case. In particular, the Sommer parameter, $r_{0}$, is ideal as it sets the scale by equating the force between two static quarks in QQCD and full QCD at a precise separation of $r_{0}=0.49$ fm. 


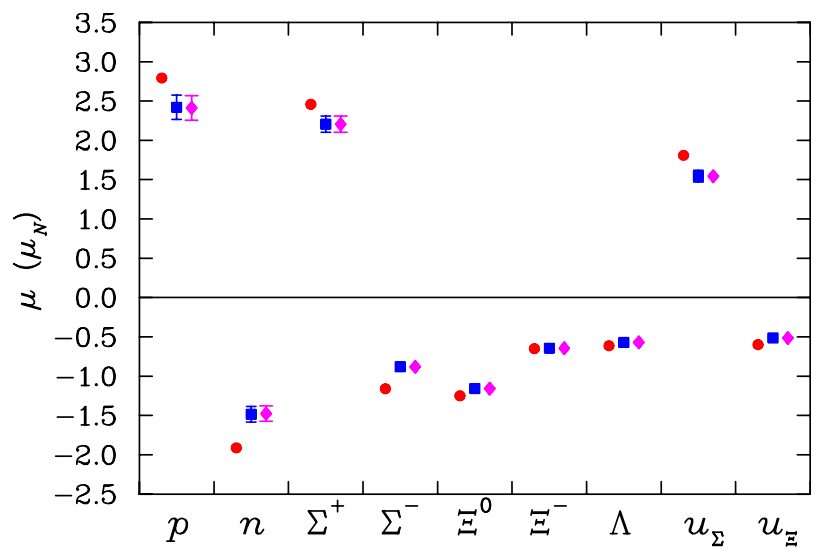

Fig. 12. The insensitivity of finite-volume quenched chiral effective field theory to NLNA decuplet-baryon contributions. Results including decuplet intermediate states in chiral effective field theory (squares) are compared with results excluding the decuplet (diamonds). Experimental measurements (circles), are illustrated at left for each baryon for reference

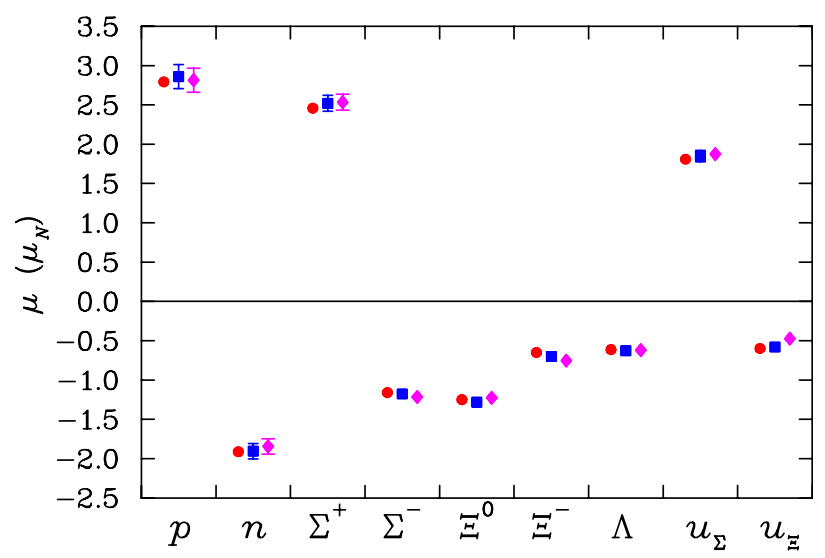

Fig. 13. The dependence of octet-baryon magnetic moments on the inclusion of NLNA decuplet-baryon contributions in the process of correcting the pion cloud of quenched chiral effective field theory. Preferred results including decuplet intermediate states in chiral effective field theory (squares) are compared with results excluding the decuplet (diamonds). Experimental measurements (circles), are illustrated at left for each baryon for reference

The string tension might also be used, but screening of the potential in full QCD makes this measure poorly defined. However, we consider it here as a measure of the systematic error encountered in setting the scale of the lattice QCD results. Figure 14illustrates this scale dependence on baryon magnetic moments and Fig. [15illustrates the rather minor impact this systematic uncertainty has on the valence-quark moment ratios vital to determining the sign of $G_{M}^{s}$.

Collecting the variation of the valence-quark moment ratios from variations in $\Lambda$ (which maintain one-standarddeviation agreement with experiment), variations in setting the lattice scale and statistical errors determined by a

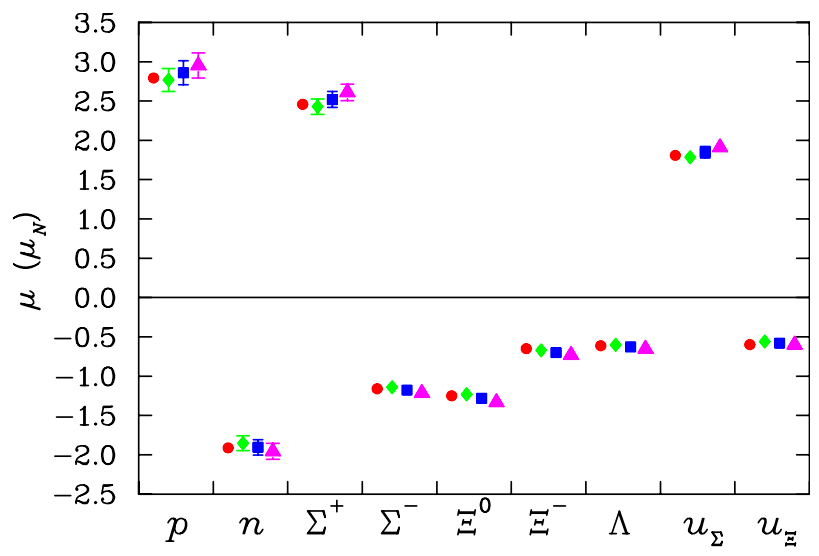

Fig. 14. The dependence of octet-baryon magnetic moments on the matching criteria for determining the lattice spacing, $a$. While $a_{r_{0}}=0.128 \mathrm{fm}$ set by $r_{0}$ (square symbols) is the preferred method for determining the scale, results for $a_{\sigma}=0.134 \mathrm{fm}$ set by the string tension (triangles) and a third spacing of $0.122 \mathrm{fm}$ (diamonds) are also illustrated. Experimental measurements (filled circles), are illustrated at left for each baryon

third-order single-elimination jackknife analysis, one finds

$$
\frac{u^{p}}{u^{\Sigma}}=1.092 \pm 0.030 \text { and } \frac{u^{n}}{u^{\Xi}}=1.254 \pm 0.124 .
$$

Using the experimental magnetic moments, one observes that (2)

$$
G_{M}^{s}=\left(\frac{{ }^{\ell} R_{d}^{s}}{1-{ }^{\ell} R_{d}^{s}}\right)\left[-1.033-\frac{u^{n}}{u^{\Xi}}(-0.599)\right],
$$

is least sensitive to variation in the valence-quark moment ratio, and hence provides the most precise determination for $G_{M}^{s}$. Figure 16 plots $G_{M}^{s}$ as a function of ${ }^{\ell} R_{d}^{s}$ with standard error limits provided by (6).

\section{Estimating ${ }^{\ell} R_{d}^{s} \equiv G_{M}^{s} /{ }^{\ell} G_{M}^{d}$}

The symmetry of the three-point correlation function [14] describing the sea-quark loop contributions to the nucleon, depicted in the right-hand side of Fig. 1, ensure that the chiral expansion for this quantity is identical for all three quark flavours, up to simple charge factors. For the $d$ - or $s$-quark loop contributions, the only difference that can arise is whether one evaluates the chiral expansion at the pion or kaon mass. The leading non-analytic contribution to the chiral expansion involves two pseudoscalar meson propagators, and therefore one expects contributions to ${ }^{\ell} R_{d}^{s}$ in the ratio $m_{\pi}^{4} / m_{K}^{4} \sim 0.1$.

To be more precise, one can use the same successful (single-parameter) model, previously used to correct the quenched simulation results to full QCD, as highlighted in Figs. 7 and 8 , to provide an estimate for ${ }^{\ell} R_{d}^{s}$. Evaluating the loop integrals with $\Lambda=0.8 \pm 0.2 \mathrm{GeV}$ yields ${ }^{\ell} R_{d}^{s}=0.139$ with $0.096 \leq{ }^{\ell} R_{d}^{s} \leq 0.181$. This uncertainty dominates the final uncertainty in $G_{M}^{s}$. 
Table 1. Sources of uncertainty and their contribution to the strangeness magnetic moment of the nucleon, $G_{M}^{s}$, in units of nuclear magnetons, $\mu_{N}$. Uncertainties are documented for $G_{M}^{s}$ obtained from the valence-quark ratio $u^{p} / u^{\Sigma}$ in (1), from the valence-quark ratio $u^{n} / u^{\Xi}$ in (2) and from a statistically weighted (SW) average of these two determinations

\begin{tabular}{llccc}
\hline Uncertainty Source & Parameter Range & $\begin{array}{c}u^{p} / u^{\Sigma}, \text { (1) } \\
G_{M}^{s}=-0.045\end{array}$ & $\begin{array}{c}u^{n} / u^{\Xi}, \sqrt{2} \text { ) } \\
G_{M}^{s}=-0.046\end{array}$ & $\begin{array}{c}\text { SW Average } \\
G_{M}^{s}=-0.046\end{array}$ \\
\hline Statistical Errors & & 0.016 & 0.009 & 0.008 \\
Chiral corrections & $0.7 \leq \Lambda \leq 0.9 \mathrm{GeV}$ & 0.001 & 0.002 & 0.002 \\
Scale Determination & $0.122 \leq a \leq 0.134 \mathrm{fm}$ & 0.001 & 0.002 & 0.002 \\
${ }^{\ell} R_{d}^{s}$ Determination & $0.096 \leq{ }^{\ell} R_{d}^{s} \leq 0.181$ & 0.016 & 0.017 & 0.017 \\
\hline Total Uncertainty & & 0.023 & 0.019 & 0.019 \\
\hline
\end{tabular}

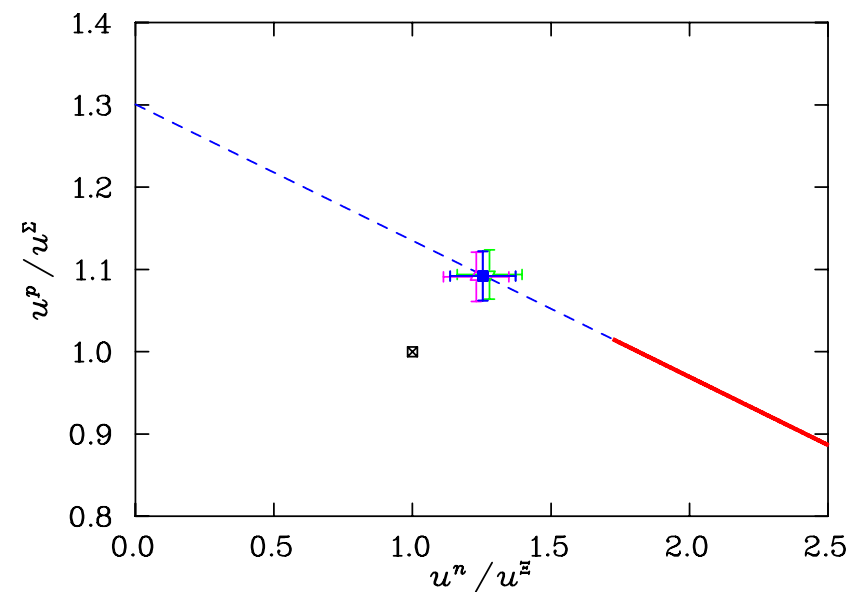

Fig. 15. The charge symmetry constraint line (dashed $G_{M}^{s}(0)<0$, solid $\left.G_{M}^{s}(0)>0\right)$ on the ratios $u^{p} / u^{\Sigma}$ and $u^{n} / u^{\Xi}$. The dependence of the ratios from chirally-corrected quenched lattice QCD on the scale parameter, $a=0.134,0.128$, and $0.122 \mathrm{fm}$, is illustrated by the cluster of points with $a$ decreasing from left to right

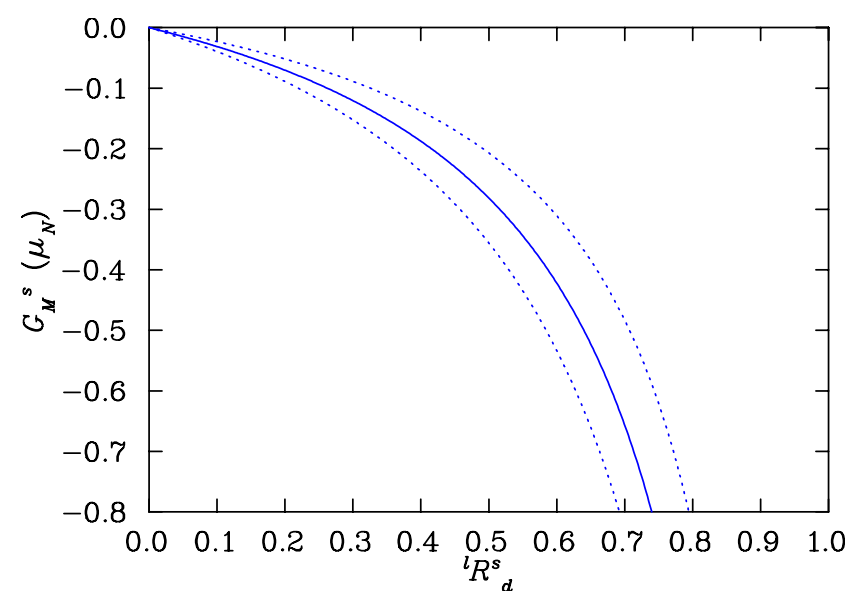

Fig. 16. The dependence of $G_{M}^{s}$ on the strange to light seaquark loop ratio ${ }^{\ell} R_{d}^{s}$. Standard error limits have there origin in the systematic error summary of 6
Table 1 summarizes the sources of uncertainty and their contributions to the final determination

$$
G_{M}^{s}=-0.046 \pm 0.019 \mu_{N}
$$

for the strange quark contribution to the magnetic moment of the nucleon.

Acknowledgements. We thank the Australian Partnership for Advanced Computing (APAC) for generous grants of supercomputer time which have enabled this project. Support from the South Australian Partnership for Advanced Computing (SAPAC) and the National Facility for Lattice Gauge Theory is also gratefully acknowledged. DBL thanks Jefferson Lab for their kind hospitality where the majority of this research was performed. This work is supported by the Australian Research Council and by DOE contract DE-AC05-84ER40150 under which SURA operates Jefferson Lab.

\section{References}

1. D.B. Leinweber et al.: arXiv:hep-lat/0406002

2. G.A. Miller, B.M. Nefkens, I. Slaus: Phys. Rept. 194, 1 (1990)

3. J.M. Zanotti et al.: Phys. Rev. D 65, 074507 (2002) [heplat/0110216]

4. D.B. Leinweber et al.: Eur. Phys. J. A 18, 247 (2003) [nuclth/0211014]

5. J.M. Zanotti et al.: hep-lat/0405015

6. J.M. Zanotti et al.: hep-lat/0405026

7. R.D. Young et al.: Prog. Part. Nucl. Phys. 50, 399 (2003) [hep-lat/0212031]

8. D.B. Leinweber, A.W. Thomas, R.D. Young: Phys. Rev. Lett. 92, 242002 (2004) [hep-lat/0302020]

9. R.D. Young, D.B. Leinweber, A.W. Thomas: arXiv:heplat/0406001

10. J.F. Donoghue, B.R. Holstein, B. Borasoy: Phys. Rev. D 59, $036002(1999)$

11. D.B. Leinweber: Phys. Rev. D 69, 014005 (2004) [heplat/0211017]

12. M.J. Savage: Nucl. Phys. A 700, 359 (2002) [nuclth/0107038]

13. R.D. Young et al.: Phys. Rev. D 66, 094507 (2002) [arXiv:hep-lat/0205017]

14. D.B. Leinweber: Phys. Rev. D 53, 5115 (1996) hep$\mathrm{ph} / 9512319$ 